\title{
CIRCLES OF CURVATURE AT POINTS OF PARABOLA IN ISOTROPIC PLANE
}

\author{
Vladimir Volenec ${ }^{1}$, Marija Šimić Horvath ${ }^{1,2, *}$ and Ema Jurkin ${ }^{1,3}$ \\ ${ }^{1}$ Department of Mathematics, University of Zagreb, Bijenička c. 30, HR-10000 Zagreb, Croatia \\ ${ }^{2}$ Faculty of Architecture, University of Zagreb, Kačićeva 26, HR-10000 Zagreb, Croatia \\ ${ }^{3}$ Faculty of Mining, Geology and Petroleum Engineering, University of Zagreb, Pierottijeva 6, HR-10000 Zagreb, \\ Croatia \\ Communicated by László Tóth \\ Original Research Paper \\ Received: May 7, 2021 - Accepted: Jul 1, 2021 \\ First published online: October 4, 2021 \\ C 2021 The Author(s)

\section{ABSTRACT} \\ The authors have studied the curvature of the focal conic in the isotropic plane and the form of the circle of curvature at its \\ points has been obtained. Hereby, we discuss several properties of such circles of curvature at the points of a parabola in the \\ isotropic plane.
}

\section{KEYWORDS}

isotropic plane, parabola, circle of curvature

MATHEMATICS SUBJECT CLASSIFICATION (2020)

Primary 51N25; Secondary 51N15

\section{INTRODUCTION}

This paper deals with the properties of the parabola in the isotropic plane concerning a circle of curvature of its points. As every circle in the isotropic plane has the absolute point for the centre, there is no need to study the centers of the circle of curvature at the points.

The curvature of the focal conic in the isotropic plane was studied in the paper [15]. Let us recall just few notions about isotropic plane.

The isotropic plane is a real projective metric plane whose absolute figure is a pair consisting of an absolute point $\Omega$ and an absolute line $\omega$ incident with it. If any point $T=\left(x_{0}: x_{1}: x_{2}\right)$ is presented in homogeneous coordinate in the projective plane, then $\Omega=(0: 1: 0)$ and the line $\omega$ with $x_{2}=0$ are chosen.

Points incident to the absolute line $\omega$ are isotropic points and lines incident to the absolute point $\Omega$ are isotropic lines.

Further on, we will mention few very well known metric quantities in the isotropic plane for which we assume that $x=\frac{x_{0}}{x_{2}}$ and $y=\frac{x_{1}}{x_{2}}$.

\footnotetext{
${ }^{*}$ Corresponding author. E-mail: marija.simic@arhitekt.hr
} 
Two lines with the same isotropic point are parallel lines and two points incident with the same isotropic line are parallel points.

A distance between two non parallel points $T_{1}=\left(x_{1}, y_{1}\right)$ and $T_{2}=\left(x_{2}, y_{2}\right)$ is defined as $d\left(T_{1}, T_{2}\right):=$ $x_{2}-x_{1}$. In the case of parallel points $T_{1}=\left(x, y_{1}\right)$ and $T_{2}=\left(x, y_{2}\right)$, a span $s\left(T_{1}, T_{2}\right):=y_{2}-y_{1}$ is defined. Both quantities are directed.

The line $p$ in the isotropic plane is given by $y=k x+l, \quad k, l \in \mathbb{R}$ and labelled by $p=(k, l)$. A distance from the point $P$ to the line $p$ is defined as the span $s(N, P)$, where $N$ is the point on $p$ parallel to $P$.

For two points $T_{1}=\left(x_{1}, y_{1}\right)$ and $T_{2}=\left(x_{2}, y_{2}\right)$ the midpoint $M$ is of the form

$$
M=\left(\frac{1}{2}\left(x_{1}+x_{2}\right), \frac{1}{2}\left(y_{1}+y_{2}\right)\right) \text {. }
$$

For two lines $p_{i}=\left(k_{i}, l_{i}\right), i=1,2$, their bisector is given by equation $y=\frac{1}{2}\left(k_{1}+k_{2}\right) x+\frac{1}{2}\left(l_{1}+l_{2}\right)$. A circle, the conic touching $\omega$ at $\Omega$, has the equation $y=u x^{2}+v x+w, u \neq 0, \quad u, v, w \in \mathbb{R}$.

Metric quantities and notions related to the geometry in the isotropic plane can be found in [13], [14] and [6].

It is shown in [2] that every focal conic in the isotropic plane, by choosing a suitable affine coordinate system can be represented by

$$
y^{2}=\epsilon x^{2}+x
$$

where $\epsilon=-1, \epsilon=0$ or $\epsilon=1$ depending on whether the conic given in (1.1) is an ellipse, a parabola or a hyperbola. This conic has the $x$-axis as its axis and one focus is $O=(0,0)$. The second focus for the ellipse and the hyperbola is of the form $O=(-\epsilon, 0)$. This conic can be parametrized as well by

$$
x=\frac{1}{t^{2}-\epsilon}, \quad y=\frac{t}{t^{2}-\epsilon},
$$

where $t \in \mathbb{R} \cup\{\infty\}$. Hence, a parabola $\mathcal{P}$ in the isotropic plane is presented in the standard form by

$$
y^{2}=x
$$

Focus, as well as the vertex of parabola is the origin $O=(0,0)$, the axis $\mathcal{A}$ of parabola is the $x$-axis and the directrix $\mathcal{D}$ is $y$-axis. The latter one is tangent to parabola at $O=(0,0)$. By replacing $t \rightarrow \frac{1}{t}$ and due to [15] the circle of curvature $\mathcal{C}$ of parabola $\mathcal{P}$ at the point $T=\left(t^{2}, t\right)$ is given by

$$
x^{2}-6 t^{2} x+8 t^{3} y-3 t^{4}=0
$$

The diameter of $\mathcal{P}$ at $T$ intersects its circle of curvature residually in the point $T^{\prime \prime}=\left(5 t^{2}, t\right)$ and for $T T^{\prime \prime}$ and $O T$ due to [15, Theorem 8] the following is valid

$$
T T^{\prime \prime}=4 O T \text {. }
$$

The same claim in the Euclidean plane can be found in [3] and [12].

\section{ON CIRCLES OF CURVATURE OF PARABOLA IN ISOTROPIC PLANE}

Further on, the properties of the circles of curvature of $\mathcal{P}$ will be discussed.

THEOREM 2.1. Let $\mathcal{P}$ be a parabola (1.3) in the standard form in the isotropic plane and let $T$ be one of its points. The circle of curvature of parabola $\mathcal{P}$ at the point $T$ intersects $\mathcal{P}$ in the point $T^{\prime}$, residually. Then the line $T T^{\prime}$ is tangent to one parabola, i.e. all such lines determine one parabola.

Motivation for this theorem is found in the Euclidean case in the question [10] and in corresponding solutions [5], [9], and [11].

Proof. To find the intersection point of (1.3) and (1.4) a calculation leads to $y^{4}-6 t^{2} y^{2}+8 t^{3} y-3 t^{4}=0$, i.e. $(y-t)^{3}(y+3 t)=0$. The solution $y=t$ is the triple intersection point $T=\left(t^{2}, t\right)$ and the solution 


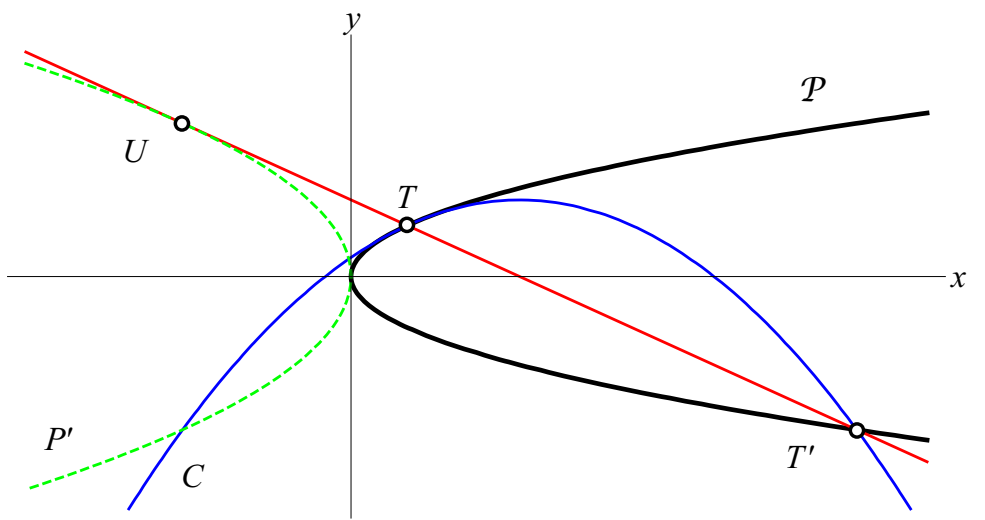

Figure 1. Theorem 2.1

$y=-3 t$ is the point $T^{\prime}=\left(9 t^{2},-3 t\right)$, the fourth intersection point of the parabola $\mathcal{P}$ and the circle of curvature $C$ at the point $T$. The line $T T^{\prime}$ has the equation

$$
y=-\frac{x}{2 t}+\frac{3 t}{2}
$$

Changing $t$, the line (2.1) touches parabola $\mathcal{P}^{\prime}$ given by

$$
y^{2}=-3 x .
$$

Namely, taking (2.1) and (2.2) and eliminating $y$, one gets $\left(\frac{x}{2 t}+\frac{3 t}{2}\right)^{2}=0$ with double solution $x=-3 t^{2}$. Hence the point of contact of parabola $\mathcal{P}^{\prime}$ and the line $T T^{\prime}$ is the point $U=\left(-3 t^{2}, 3 t\right)$.

One intersection point of parabolas $\mathcal{P}$ and $\mathcal{P}^{\prime}$ is the point $U^{\prime}=\left(-3 t^{2},-3 t\right)$. The midpoint of points $T$ and $T^{\prime}$ is the point $M=\left(5 t^{2},-t\right)$ which obviously determines the parabola $\mathcal{P}^{\prime \prime}$ given by $y^{2}=\frac{1}{5} x$. The same parabola is determined by the point $T^{\prime \prime}$ as well, the point simetric to $M$ with respect to the axis $\mathcal{A}$ of parabola $\mathcal{P}$. Points $T$ and $U^{\prime}$ are incident with the line $y=\frac{1}{t} x$ passing through the origin $O$. The midpoint of $T^{\prime}$ and $U$ is the point $V^{\prime}=\left(3 t^{2}, 0\right)$ incident to the axis $\mathcal{A}$ of parabola $\mathcal{P}$.

The fact that $T T^{\prime}$ determines a parabola can be found in Euclidean case in [1].

The tangent $\mathcal{T}$ at $T$ of the parabola $\mathcal{P}$ is given by $y=\frac{x}{2 t}+\frac{t}{2}$. Due to [15] lines $\mathcal{T}$ and $T T^{\prime}$ are antiparallel with respect to the axis $\mathcal{A}$ of parabola $\mathcal{P}$.

Analogous result in Euclidean plane can be found in [7]. The tangent $\mathcal{T}$ intersects the axis of parabola $\mathcal{P}$ in the point $\left(-t^{2}, 0\right)$. The result in the Euclidean case given in [4] is valid in isotropic plane as well. Namely, the line $T T^{\prime}$ intersects the vertex tangent, i.e. directrix, of the parabola $\mathcal{P}$ at the point $V=\left(0, \frac{3}{2} t\right)$. Lines $O T$ and $O U$ form a harmonic quadraple of lines with its bisector, the axis of parabola, and the vertex tangent. Hence, the pairs of points $T, U$ and $V, V^{\prime}$ are harmonic points.

The tangent $\mathcal{T}$ intersects directrix $x=0$ at the point $V^{\prime \prime}=\left(0, \frac{1}{2} t\right)$, so the equality $O V=3 O V^{\prime \prime}$ holds. The analogous Euclidean case is given in [4]. Hereby, the claim is telling the following:

THEOREM 2.2. The span from the vertex of parabola $\mathcal{P}$ to the tangent of the same parabola at the point $T$ is $\frac{1}{3}$ of the span from the vertex of the parabola to the common chord of parabola and the circle of curvature of the point $T$.

The result given in [8] is valid in the isotropic plane as well saying: 
THEOREM 2.3. The radical axis of the circles of curvature at the endpoints of any chord conjugate to a given diameter of a parabola passes through a fixed point. Furthermore, the locus of this point for different diameters is a parabola.

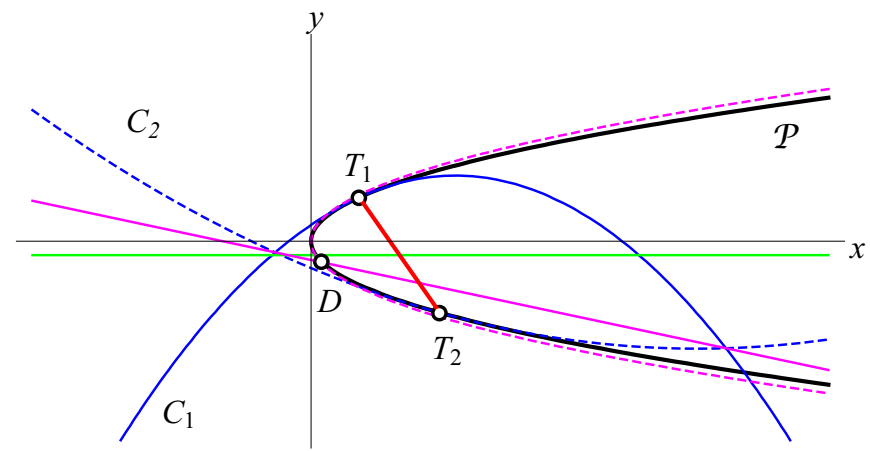

Figure 2. Theorem 2.3

Proof. Let $T_{1}=\left(t_{1}{ }^{2}, t_{1}\right)$ and $T_{2}=\left(t_{2}{ }^{2}, t_{2}\right)$ be two different points of the parabola $\mathcal{P}$, and $\mathcal{C}_{1}$ and $\mathcal{C}_{2}$ circles of curvature at those points. If we take $t \rightarrow t_{1}$ and $t \rightarrow t_{2}$ in (1.4) and then subtract these two equations, we get the radical axis $\mathcal{P}_{12}$ of $\mathcal{C}_{1}$ and $\mathcal{C}_{2}$ with equation

$$
6\left(t_{1}+t_{2}\right) x-8\left(t_{1}^{2}+t_{1} t_{2}+t_{2}^{2}\right) y+3\left(t_{1}^{2}+t_{2}^{2}\right)\left(t_{1}+t_{2}\right)=0 .
$$

A diameter $\mathcal{D}$ of parabola $\mathcal{P}$ that is conjugate to the line $T_{1} T_{2}$ is given with $y=t$, where $t=\frac{1}{2}\left(t_{1}+t_{2}\right)$. The equation (2.3) turns to

$$
3 t x-\left(8 t^{2}-2 t_{1} t_{2}\right) y+6 t^{3}-3 t t_{1} t_{2}=0 .
$$

With given diameter $\mathcal{D}$, the locus of all the radical axes $\mathcal{P}_{12}$ with variable (mutually parallel) lines $T_{1} T_{2}$ has the equation (2.4) and it is obviously incident to the fixed point $D=\left(2 t^{2}, \frac{3}{2} t\right)$. It is easy to prove that the point $D$ with variable $t$ determines a parabole given by $y^{2}=\frac{9}{8} x$.

The intersection point of the line (2.3) and the directrix $x=0$ of parabola $\mathcal{P}$ has ordinate

$$
y=\frac{3\left(t_{1}+t_{2}\right)\left(t_{1}{ }^{2}+t_{2}{ }^{2}\right)}{8\left(t_{1}^{2}+t_{1} t_{2}+t_{2}^{2}\right)} .
$$

If $T_{3}=\left(t_{3}{ }^{2}, t_{3}\right)$ denotes some third point of $\mathcal{P}$, and $\mathcal{C}_{3}$ denotes its circle of curvature, then the radical axis $\mathcal{P}_{13}$ of circles $\mathcal{C}_{1}$ and $\mathcal{C}_{3}$ intersects the directrix of the parabola $\mathcal{P}$ in the point having ordinate

$$
y^{\prime}=3 \frac{\left(t_{1}+t_{3}\right)\left(t_{1}^{2}+t_{3}^{2}\right)}{8\left(t_{1}^{2}+t_{1} t_{3}+t_{3}^{2}\right)} .
$$

Ordinates (2.5) and (2.6) are equal under the assumption

$$
t_{1} t_{2} t_{3}\left(t_{1}+t_{2}+t_{3}\right)+t_{1}{ }^{2} t_{2}{ }^{2}+t_{1}{ }^{2} t_{3}{ }^{2}+t_{2}{ }^{2} t_{3}{ }^{2}=0 .
$$

Taking notations $t_{1}+t_{2}=s$ and $t_{1} t_{2}=p$, (2.7) can be written in the form

$$
p^{2}+t_{3}^{2}\left(s^{2}-p\right)+t_{3} p s=0
$$

as well, so we get

$$
t_{1}^{2}+t_{1} t_{2}+t_{2}^{2}=s^{2}-p=\frac{-p}{t_{3}^{2}}\left(p+t_{3} s\right)=\frac{-p}{t_{3}^{2}}\left(t_{1} t_{2}+t_{1} t_{3}+t_{2} t_{3}\right),
$$

and

$$
t_{1}^{2}+t_{2}^{2}=s^{2}-2 p=\frac{-p}{t_{3}^{2}}\left(p+t_{3} s\right)-p=\frac{-p}{t_{3}^{2}}\left(p+t_{3} s+t_{3}{ }^{2}\right)=\frac{-p}{t_{3}{ }^{2}}\left(t_{1}+t_{3}\right)\left(t_{2}+t_{3}\right) .
$$


So, out of (2.5) it follows

$$
y=\frac{3\left(t_{1}+t_{2}\right)\left(t_{1}+t_{3}\right)\left(t_{2}+t_{3}\right)}{8\left(t_{1} t_{2}+t_{1} t_{3}+t_{2} t_{3}\right)}
$$

We have proved the following theorem that is new result in the isotropic plane. The motivation for it was found in the result of [16] that can't be turned into the isotropic plane.

THEOREM 2.4. The radical center of circles of curvature for three points $T_{1}=\left(t_{1}{ }^{2}, t_{1}\right), T_{2}=\left(t_{2}{ }^{2}, t_{2}\right)$ and $T_{3}=\left(t_{3}{ }^{2}, t_{3}\right)$ of the parabola $\mathcal{P}$, is incident to its directrix under assumption (2.7) and then it has the ordinate given by (2.8).

\section{REFERENCES}

[1] E. N. BARISIEn. Question 835 d'examen, Mathesis, 8(2):102-103, 1898.

[2] J. Beban-Brkić, M. Šimić And V. Volenec V. On Foci and Asymptotes of Conics in Isotropic Plane. Sarajevo f. Math., 3(16):257-266, 2007.

[3] A. Cayley. Question 1771, Educ. Times, 4:70-71, 1865.

[4] R. H. Graves. On the chord common to a parabola and the circle of curvature at any point. Ann.of Math., 3:50, 1887.

[5] J. HaAg. Solution of Question 644. Nouv. Ann. Math., 2(2) (1863), 415-419.

[6] R. Kolar-Šuper, Z. Kolar-Begović, V. Volenec and J. Beban-Brkić. Metrical relationships in standard triangle in an isotropic plane. Math Commun, 10 (2005), 159-167.

[7] K. Maск. Ueber die Krümmungskreise der Parabel. Arch. Math. Phys., 61 (1877), 385-406.

[8] M. A. NAsh. Question 6602. Educ. Times, 37 (1882), 37-38.

[9] E. D’Ovidio. Solution of Question 644. Giorn. Mat., 1:285-286, 1836.

[10] Question 644. Nouv. Ann. Math., 2(2):94, 1863.

[11] G. Recchia. Solution of Question 644. Giorn. Mat., 1:160, 1836.

[12] E. Rouché And C. De Comberousse. Traité de géométrie, 8. éd., Gauthier-Villars, Paris 1912.

[13] H. SACHs. Ebene isotrope Geometrie. Braunschweig/Wiesbaden, Germany: Vieweg-Verlag, 1987.

[14] K. Strubecker. Geometrie in einer isotropen Ebene. Math Naturwiss Unterr, 1962-63(15):297306, 343-351, 385-394.

[15] M. Šimić, V. Volenec AND J. Beban-Brkić. Curvature of the focal conic in the isotropic plane. Sarajevo f. Math., 8(18):117-123, 2010.

[16] R. Tucker. Question 8693. Educ. Times, 48:112-113, 1887.

Open Access statement. This is an open-access article distributed under the terms of the Creative Commons AttributionNonCommercial 4.0 International License (https://creativecommons.org/licenses/by-nc/4.0/), which permits unrestricted use, distribution, and reproduction in any medium for non-commercial purposes, provided the original author and source are credited, a link to the CC License is provided, and changes - if any - are indicated. 